\title{
New Insights: PET Imaging to Document Synergistic Therapy Effect of the "Old," Ancient Recipe and the "New," Modern Drug in Stroke
}

\author{
A. Cahid Civelek ${ }^{1,2}$
}

${ }^{I}$ Department of Radiology and Imaging Sciences, Body Imaging-MRI, National Institutes of Health, Bethesda, Maryland; and

${ }^{2}$ Division of Nuclear Medicine, The Johns Hopkins Medical Institutions, Baltimore, Maryland

$\mathbf{S}$ number one cause of disability in the United States (1). Although prevention and effective treatment of stroke is of utmost importance, effective treatments to promote tissue repair and functional recovery after stroke are lacking. However, recent advances in stem cell biology have raised expectations that consequence of cerebral ischemia may be improved by the transplantation of stem cells and their progeny originating from various sources. Subsequently, local or systemic delivery of neuronal stem cells (NSCs) or induced pluripotent stem cells (iPSCs) has emerged as a recovery-promoting approach in preclinical animal models (2).

Currently in China, ancient recipes and practices of traditional Chinese medicine (Chinese patent medicine) are regularly used as a complementary therapeutic strategy in the care of stroke patients both in Western medical hospitals and in traditional Chinese medicine hospitals (3).

In the November 2015 issue of The Journal of Nuclear Medicine, Zhang et al. presented their novel findings on dynamic changes of cerebral metabolism and neurologic function after stem cells (iPSCs or NSCs) and Chinese patent medicine applications in a rat model of cerebral ischemia-reperfusion injury (4). They conducted serial ${ }^{18} \mathrm{~F}$ FDG PET imaging to investigate the dynamic metabolic changes after combined therapeutic approach of stem cells (iPSCs or NSCs) and Chinese patent medicine. After individual or combination treatment with stem cells and Chinese medicine (Qing-Kai-Ling [QKL]), a significant increase in cerebral metabolism and improvement in neurologic functions were found. Transplanted iPSCs and NSCs survived and differentiated into mature neuronal cells, astrocytes, or endothelial cells. In addition, by direct comparison among different treatment groups, they observed different time course patterns of glucose metabolic recovery: NSCs had a significantly better effect within the first $2 \mathrm{wk}$, whereas iPSCs had a more steady effect over the course of $2 \mathrm{wk}$. The iPSCs + QKL group showed a steady increase of ${ }^{18} \mathrm{~F}-\mathrm{FDG}$ accumulations in the infarct zone and corresponding neurofunctional recovery during/after the 4-wk period. Neurofunctional tests and PET imaging indicated steady

Received Oct. 20, 2015; revision accepted Oct. 22, 2015

For correspondence or reprints contact: A. Cahid Civelek, University of Louisville Medical Center, 530 S. Jackson St., Suite C07, Louisville, KY 40202.

E-mail: accivelek@gmail.com

Published online Nov. 5, 2015.

COPYRIGHT (C) 2016 by the Society of Nuclear Medicine and Molecular Imaging, Inc.

DOI: $10.2967 /$ jnumed.115.166702 recovery with the use of Chinese medicine (QKL) during the duration of treatment.

Interestingly, in this study, PET was applied to test the accuracy of traditional oriental medicine, which might be regarded as the bedrock of science and medicine. Their results, perhaps for the first time, provided new and important insights into the underlying mechanism of a combined approach of stem cell and traditional Chinese medicine.

Although stem cell-based therapy holds great promise for the therapy outcome of treatment-refractory diseases, including stroke, recent clinical studies have not shown sufficient efficacy required for their use as a first-line therapy. This may partially be due to the undetermined fate of cells. Therefore, real-time cell trafficking and monitoring of the responses to therapies are essential to validate the full potential of stem cell-based therapy. Stem cells are often detected in vivo through the use of retroviruses or with thymidine or bromdodeoxyuridine labeling. Retroviruses infect only dividing cells, can be passed on to all progeny of the infected cells, and reflect a particular cell's lineage (5). However, currently this procedure is generally inefficient and not widely applicable. Recent studies have shown how real-time in vivo molecular imaging may help in identifying hurdles toward clinical translation and designing potential strategies that may contribute to successful transplantation of stem cells and their improved outcomes. ${ }^{18}$ F-FDG PET imaging technology is an ideal, costeffective, and efficient technique for in vivo monitoring of the posttherapy outcomes of the diseases, which is widely available in routine clinical practice.

The co-use of ancient recipes and practices of traditional Chinese medicine (Chinese patent medicine) with western medicine is not new. Over the past decades, Chinese medical scientists and pharmaceutical companies have focused on traditional Chinese medicines and tried to isolate their active ingredients with the intent to optimize their individual utilizations.

This year's Nobel Prize in Physiology or Medicine 2015 was divided, one half jointly to William C. Campbell and Satoshi Komura (for their discoveries concerning a novel therapy against infections caused by roundworm parasites) and the other half to Youyou Tu, a female Chinese scientist, for her discoveries concerning a novel therapy against Malaria. Her rediscovery of artemisinin (Qinghaosu) and its use in the treatment of malaria and more so for her own unique technique of improved extraction method of the active ingredient of the plant resulted in a medical advance that has saved millions of lives across the globe, especially among the developing world's poorest children (6-8). 
Most remedies in traditional Chinese medicine are compound formulae or mixtures that contains many species of herbs and varieties of chemicals (7). In December 2006, the U.S. Food and Drug Administration issued a draft guidance for the industry-complementary and alternative medicine products and their regulation (9).

In vivo molecular imaging by depicting different biochemical processes may provide a measure of the entire body's response to a complex mixture of herbs or medicines. The study by Zhang et al. (4) in the November $2015 J N M$ has explored an alternative and complementary therapy in a stroke model and demonstrated feasibility and efficacy of PET imaging for monitoring functional and metabolic recovery after complementary cellular and herbalbased medicine. Given the enthusiasm in the research community on stem cell and oriental therapies (ancient recipes), it is likely that new research and observations using molecular imaging will rapidly be replicated, and knowledge on potentials of synergistic effects of western and oriental medicine will be further extended.

It seems as if the synergism between the ancient recipe (old) and the modern drug (new) and the East and the West should be further explored.

\section{DISCLOSURE}

No potential conflict of interest relevant to this article was reported.

\section{REFERENCES}

1. Mozaffarian D, Benjamin EJ, Go AS, et al. Heart disease and stroke statistics: 2015 update-a report from the American Heart Association. Circulation. 2015;131:e29-e322.

2. Pluchino S, Peruzzotti-Jametti L. Rewiring the ischaemic brain with human-induced pluripotent stem cell-derived cortical neurons. Brain. 2013;136:3525-3527.

3. Wu B, Liu M, Liu H, et al. Meta-analysis of traditional Chinese patent medicine for ischemic stroke. Stroke. 2007;38:1973-1979.

4. Zhang H, Song F, Xu C, et al. Spatiotemporal PET imaging of dynamic metabolic changes after combined therapeutic approach of induced pluripotent stem cells, neuronal stem cells, and Chinese patent medicine in a rat model of cerebral ischemia-reperfusion injury. $J$ Nucl Med. 56:1774-1779.

5. Gage FH. Mammalian neural stem cells. Science. 2000;287:1433-1438.

6. Tu Y. The discovery of artemisinin (qinghaosu) and gifts from Chinese medicine. Nat Med. 2011;17:1217-1220.

7. Qiu J. Traditional medicine: a culture in the balance. Nature. 2007;448:126-128.

8. Miller LH, Su X. Artemisinin: discovery from the Chinese herbal garden. Cell. 2011;146:855-858.

9. Complementary and alternative medicine products and their regulation by the Food and Drug Administration. U.S. Food and Drug Administration website. http://www.fda.gov/RegulatoryInformation/Guidances/ucm144657.htm. December 2006. Accessed November 16, 2015 